\title{
Approaches to measuring academic resilience: A systematic review
}

Tudor, Kate Elizabeth $\triangle$

Loughborough University, London, UK (k.e.tudor@lboro.ac.uk)

Spray, Christopher Mark

Loughborough University, London,UK (c.m.spray@lboro.ac.uk)

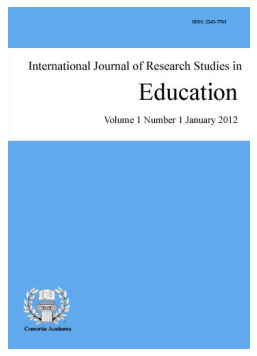

ISSN: 2243-7703 Online ISSN: 2243-7711

Received: 23 July 2017

\section{Abstract}

Recent years have seen increased government funding into resilience-building programmes in schools. However, practitioners are unable to assess the efficacy of interventions due to the lack of an available measure of academic resilience. The aim of this review is to provide an overview of the methods investigators have employed to measure academic resilience. A computerised literature search was conducted to identify journal articles where academic resilience was either; a) inferred through assessment of risk and positive adaptation or b) assessed using a measurement scale comprising protective factors. Results demonstrated significant variability in the factors utilised to represent risk and positive adaptation, and an inconsistent use of measurement scales. Different approaches to measuring academic resilience across studies leads to inconsistencies when estimating prevalence of the concept and the impact of resilience-based interventions. A discussion of the psychometric rigor of approaches to assessment is provided, with specific recommendations for future development of a measurement of academic resilience.

Keywords: academic resilience; academic buoyancy; measurement; school-based; intervention; systematic review 


\section{Approaches to measuring academic resilience: A systematic review}

\section{Introduction}

Academically resilient students have been described as those who achieve success in school despite experiencing stressful events that place them at risk of performing poorly (Wang, Haertal, \& Walberg, 1994). Research into resilience has increased substantially over recent decades and the concept is receiving more interest from politicians and scholars, with an increase demand to introduce resilience building programs as part of the national curriculum in the UK (Schofield \& Bates, 2016) and globally (Hart \& Heaver, 2015). Furthermore, under new government initiatives, trainee teachers will soon be taught how to build character and resilience, so that pupils are better equipped with the adversities they face (Schofield \& Bates, 2016). We begin this review by providing an overview of the conceptualization of academic resilience, the issues surrounding measurement of the concept and the implication this has for evaluating school-based resilience interventions.

\subsection{What is academic resilience?}

The term resilience refers to findings that some individuals have relatively good psychological outcomes, despite exposure to acute or chronic stressors that are associated with negative outcomes (Luthar, Cicchetti, \& Becker, 2000; Rutter, 2006) Over the past two decades, various definitions of resilience have been proposed, with notable discrepancies across the literature (see, for a review, Sarkar \& Fletcher, 2013). Despite the definitional and conceptual discrepancies, most definitions are based around two core concepts: adversity and positive adaptation (Windle, Bennet, \& Noyes, 2011). As the field of resilience has developed, researchers have begun to identify specific characteristics of individuals who thrive while living through significant trauma (Rutter, 1985). Identifying assets that moderate an individual's ability to respond to, and reduce the effects of, an adversity has therefore been central to examining resilience (Windle et al., 2011). These assets have also been described as 'protective factors', that is, "influences that modify, ameliorate or alter a person's response to some environmental hazard" (Rutter, 1985, p. 600). Thus, it has been proposed that measures of resilience should assess three defining components: adversity, positive adaptation and protective factors (Sarkar \& Fletcher, 2013). Moreover, due to the distinct nature of these three concepts, scholars should measure and analyse these three components separately to gain a complete picture of resilience.

The measurement of resilience is inherently influenced by conceptual issues (Windle et al., 2011), specifically whether resilience is conceptualised as a trait or process. When conceptualising resilience as a trait, researchers posit that it is the positive role of individual characteristics that enable a positive response to adversity (Rutter, 1987). Individual protective factors identified in the literature include: positive affect, self-esteem, extraversion, social support, and optimism (see, for a review, Rutter, 1985; Sarkar \& Fletcher, 2013). In contrast, researchers have viewed resilience as a process that changes over time and context, putting greater emphasis on the interaction between individuals and their environments (Luthar et al., 2000). Luthar and colleagues state that viewing resilience as a "dynamic process" accounts for the fact individuals' resilience may vary across contexts and over time. Emphasising the interaction between the individual and their environment highlights the fact that protective factors can include not only individual characteristics but also the social environment (e.g. family, peer or community support; Masten, 2001). Conceptualising resilience as a process provides a basis for the development of interventions that promote both individual characteristics and protective aspects of the social environment. The increasingly popular conception of resilience as a process that varies across time and context has prompted researchers to focus on understanding resilience in specific contexts. In light of these contextual differences, educational researchers have begun to apply the traditional concept of resilience to an academic setting.

In the academic context, resilience is defined as "the heightened likelihood of success in school and other 
life accomplishments despite environmental adversities brought about by early traits, conditions and experiences" (Wang et al., p. 46). Investigated within the framework of risk and resilience, researchers seek to identify factors that enable at-risk students to 'overcome the odds' and achieve academic success. 'Risks' (or adversity) have been defined as individual or social factors that are associated with a greater likelihood of poor development outcomes (Garmezy \& Masten, 1986). For example, Overstreet and Braun (1999) focussed on students with a low socio-economic status (SES), while other studies have investigated children from minority ethnic groups (Gonzalez \& Padilla, 1997). 'Positive adaptation' refers to success at meeting stage salient tasks (Luthar et al., 2000) and, within academic resilience, refers to academic achievement relative to the risk posed. Much like the traditional resilience literature, researchers aim to identify protective factors that moderate students' ability to respond to, and reduce the academic effects of a given risk. Individual protective factors of academically resilient students include high self-esteem, self-efficacy and autonomy (Wang et al., 1994), engagement in school (Finn \& Rock, 1997) and value in school (Perez, Espinoza, Ramos, Coronado, \& Cortes 2009). Environmental factors have also been identified that serve to protect students from the impact of risk including, parent involvement (La Foret, Watt, Diaz, McCullough, \& Barrrueco, 2000), social ties at school (Langenkamp, 2010) and, classroom environment (Samel, Sondergeld, Fischer, \& Patterson, 2011).

\section{2 'Everyday resilience': academic buoyancy}

Research into academic resilience sheds light about particular groups at risk of adversity, however, it provides limited information about how resilient the majority of students are when faced with the challenges associated everyday school life. The majority of students face less extreme, but nonetheless problematic academic challenges (Martin \& Marsh, 2008). To address this gap, Martin and Marsh (2008) introduced the concept of academic buoyancy, which refers to students' ability to "successfully deal with academic setbacks and challenges that are typical of the ordinary course of school life" (Martin \& Marsh, 2008, p. 54). Martin and colleagues proposed detailed examples of how academic buoyancy and academic resilience may be operationally differentiated. For example, while resilience refers to responses to extreme adversities (e.g., chronic underachievement or poverty), buoyancy addresses everyday stressors, or hassles, at school (e.g., patches of poor performance or pressures of competing deadlines). Moreover, academic resilience may be relevant to disengagement from school and severe affective responses (e.g., depression and anxiety), whereas buoyancy relates to periods of decreased motivation and engagement and low level affective outcomes (Martin \& Marsh, 2008).

Buoyancy therefore reflects an 'everyday resilience' that is more relevant for the majority of students who deal with the challenges of school life. The ability to successfully deal with daily school-based setbacks is likely to be influenced by multiple interconnecting factors. Martin and Marsh (2006) have suggested a number of motivational predictors of academic buoyancy, known as the 5Cs: Confidence (self-efficacy), Co-ordination (planning), Control (low uncertain control), Composure (low anxiety) and Commitment (persistence). The 5Cs have been found to predict academic buoyancy and to partially mediate between baseline and follow up academic buoyancy scores (Martin, Colmar, Davey, \& Marsh, 2010). Empirical work has begun to test the distinct nature of buoyancy and resilience. Martin (2013) reports data suggesting that academic buoyancy and academic resilience are distinct constructs, with buoyancy salient in predicting 'low level' negative academic outcomes (anxiety, uncertain control) and resilience predicting 'high-level' outcomes (self-handicapping, disengagement). Furthermore, academic buoyancy has been shown to predict academic resilience, providing preliminary evidence for an ordering effect of buoyancy and resilience. That is, individuals high in academic buoyancy may be better equipped at dealing with more extreme adversities should they occur (Martin, 2013).

The 5Cs represent a basis for the development and implementation of school-based interventions aimed at promoting students' everyday resilience to relatively minor, but significant, daily setbacks. However, Martin (2013) notes limitations with the approach to measuring academic resilience and buoyancy in this study, stating that the similarity of the measures may lead to potential bias in participants' interpretation and responses. Such observations further support a need to develop a reliable and valid measure of academic resilience and buoyancy 
in school students. There has been no intervention based on the concept of academic buoyancy to date, and it is vital to be clear on the conceptualisation and measurement before development begins to ensure an intervention is targeting the correct concept.

\subsection{School-based resilience interventions}

School-based approaches aimed at fostering resilience promote problem-solving skills, perseverance, and a positive emotional and behavioral attitude towards hard work in the face of failure. Interventions include targeting protective factors, including: individual assets (problem solving, sense of purpose, self-esteem); interpersonal factors (empathy, social competence); friends and family factors (family connectedness and positive peer relationships) and community factors (school / community connectedness). Hart and Heaver (2015) provide a comprehensive overview of school-based interventions for educational professionals to purchase and implement within their schools. It is disappointing that often, such costly interventions are implemented with little understanding of the concept, with various terminologies being used interchangeably. For example, some resilience approaches claim to be resilience based, however describe and target the more general concept of wellbeing (Hart \& Heaver, 2013). Similarly, practitioners and politicians frequently use terms such as 'resilience', 'grit' and 'persistence' interchangeably. Not only do these concepts represent different characteristics, neither may be an appropriate label for what is being promoted in a given intervention (Smith, 2015).

Moreover, interventions lack a measurement strategy to evaluate their effectiveness in targeting and promoting resilience. For example, the UK's largest school-based intervention, the UK Resilience Program, based on the Penn Resiliency Program (Gillham et al., 2007) utilized outcome measures that were inconsistent with the concepts targeted in the intervention. The evaluation of such interventions and policies requires reliable and valid measures of academic resilience, as different approaches to measuring academic resilience across studies leads to inconsistencies when estimating prevalence. This makes it difficult to assess the effectiveness of resilience-based interventions, and even more difficult for professionals to make an informed decision regarding the purchase of such interventions. Establishing a reliable and valid approach to measuring academic resilience will have implications for both the development and evaluation of resilience-based interventions.

\subsection{How do scholars measure academic resilience?}

Currently, there is no 'gold standard' measure of academic resilience. The conceptual issues that characterise the traditional resilience research (e.g. trait versus process) described above, also apply in the academic context. Most researchers to date have not measured academic resilience directly; rather resilience is inferred based on the presence of an adversity or risk, with the demonstration of positive adaptation. Measuring academic resilience this way can be carried out through 'variable-focussed' or 'person-focussed' approaches.

In variable-focussed studies, researchers test for linkages between measures of risk, positive adaptation and the role of protective factors in mediating or moderating the impact of risk on subsequent outcomes (Masten, 2001). In such models, the main effects reflect the independent influence of a protective factor to the course of the outcome (positive adaptation). Furthermore, tests of mediated effects can be performed to determine whether altering the level of a protective factor (e.g. parental involvement in school) can contribute to positive outcomes. For example, Abel (2013) tested the mediating role of perceived discrimination of African American students at-risk of poor academic performance on their subsequent Grade Point Averages. Finally, variable focussed analyses can incorporate interaction models to test for protective factors that moderate the impact of an adversity on positive outcomes, that is, decrease the impact of the adversity on positive adaptation. As stated above, the definition of a protective factor is one that "ameliorates" or "alters" a person's response to an adversity. Therefore, it is essential that protective factors are tested for their mediating or moderating role in the relationship between risk and positive adaptation. 
Person-focussed approaches to assessing academic resilience have involved the comparison of two groups of individuals, taken from the same high risk sample, who demonstrate adaptive or maladaptive outcomes (Masten, 2001). Statistical analysis, for example cluster analysis or discriminant function analysis, can then be employed to compare differences in the resilient (those who demonstrate positive adaptation) and non-resilient groups (Masten, 2001). For example, Finn and Rock (1997) compared resilient (school completers) and non-resilient (school 'drop-outs') students on measures of self-esteem and engagement. Whether researchers adopt a person-focussed or variable-focussed approach, it is important that the appropriate statistical analysis is used to understand relationships between adversity, positive adaptation and the role that protective factors can play.

A number of scales have been developed to assess psychological resilience in adult (Connor \& Davidson, 2006) and adolescent (Ungar \& Leibenberg, 2011) populations. The most psychometrically sound measures of the traditional concept of resilience (see, for a review, Windle et al., 2011) are those where the items reflect a collection of protective factors that facilitate resilience (e.g. Connor-Davidson Resilience Scale, Connor \& Davidson, 2003; Child and Youth Resilience Measure, Ungar, \& Leibenberg, 2011). As academic resilience is characterised by the similar conceptual issues, academic researchers can use the lessons learnt from the psychological resilience literature. Currently, researchers utilise a variety of measurement scales to assess academic resilience, resulting in inconsistencies in terms of the prevalence of academic resilience, leading to the question of whether researchers are truly measuring the same concept. Moreover, evaluation of interventions designed to promote academic resilience require reliable measures to ensure the appropriate concepts are targeted. The aim of the current study was to provide some coherence to the academic resilience literature by:

$>$ Providing an overview of methodologies employed to assess academic resilience. The review focuses on studies investigating academic resilience that should inform the development of school-based resilience interventions.

$>$ Reviewing the indicators of risk and positive adaptation employed to infer academic resilience. Moreover the statistical analysis utilized to determine the contributing role of protective factors will be reported.

$>$ Reviewing the psychometric rigor of measurement scales utilized to assess academic resilience.

$>$ Providing recommendations for the appropriate measure of academic resilience, in light of the psychometric lessons learned in the psychological resilience literature.

\section{Method}

\subsection{Search strategy}

In October 2016, a computerized literature search of Web of Science, PubMED, PsychINFO, ERIC and SportsDISCUS was conducted. Search strategies were built around four groups of key words: education (e.g. education*, academic*, adolescen*), resilience (e.g. resilien*, buoyan*) and measurement (e.g. scale, measure*, instrument and assess*). Asterisks were placed to account for more than one appropriate word (e.g. resilient and resilience). The search terms were also entered into Google Scholar and reference lists of previous reviews of the literature were hand searched. The search was limited to English language papers, specifically dealing with academic resilience, and or, buoyancy. Figure 1 depicts the literature retrieval process. The original search identified 2893 papers, commentaries and, reviews of the literature. The titles and abstracts of the identified papers were initially screened, and articles that did not address academic resilience or buoyancy were excluded. The full texts of the remaining articles were screened to identify articles that met the inclusion criteria.

Inclusion criteria - The focus of the search was to identify peer reviewed journal articles where academic resilience was the key focus, where the authors had engaged with the resilience evidence-base in their rationale 
and investigated resilience in the academic context. Specifically, articles were included if an attempt was made to measure student resilience through: a) the assessment of risk, positive adaptation and protective factors or b) assessment using a measurement scale. As the focus of the review was on academic resilience in school, studies of students in full-time education (aged 4- 19) were included.

Exclusion criteria - Papers not published in English were excluded from this paper if no translated version was available. Papers were excluded if only the title was available and the authors were unable to obtain a full version. Articles that addressed academic resilience in University populations were excluded. Studies that claimed to measure resilience, however, did not complete adequate assessment (see inclusion criteria), were excluded.

Data extraction - A data extraction tool was developed, adapted from a previous systematic review (viz, Simpson et al., 2014). Detailed information was extracted from each article including sample characteristics (sample size, age, gender, socio-economic status, ethnicity and 'risk status' if applicable), country of study, study design, resilience (and/ or buoyancy) measure, measure of positive adaptation, measure of protective factor(s) and the details of statistical analysis. Two reviewers discussed criteria for inclusion in the review, agreeing that articles should be included based on authors' attempts to measure resilience within an academic setting. Both reviewers have sound knowledge of the conceptualisation and theories of academic resilience. Specifically, broad screening (i.e. screening of titles and abstracts) was conducted by the first author. Working independently, the reviewers carried out narrow screening (i.e. screening of full texts) and any ambiguity with inclusion and exclusion was discussed and resolved. Data extraction was carried out by the first author, corroborated by the second author following data extraction of a sample of articles.

\section{Results}

As demonstrated in Figure 1, 47 studies included in the current review assessed academic resilience by measuring student risk (or adversity), positive adaptation and one or more protective factors. Thirty-four studies utilised measurement scales to assess academic resilience (or academic buoyancy).

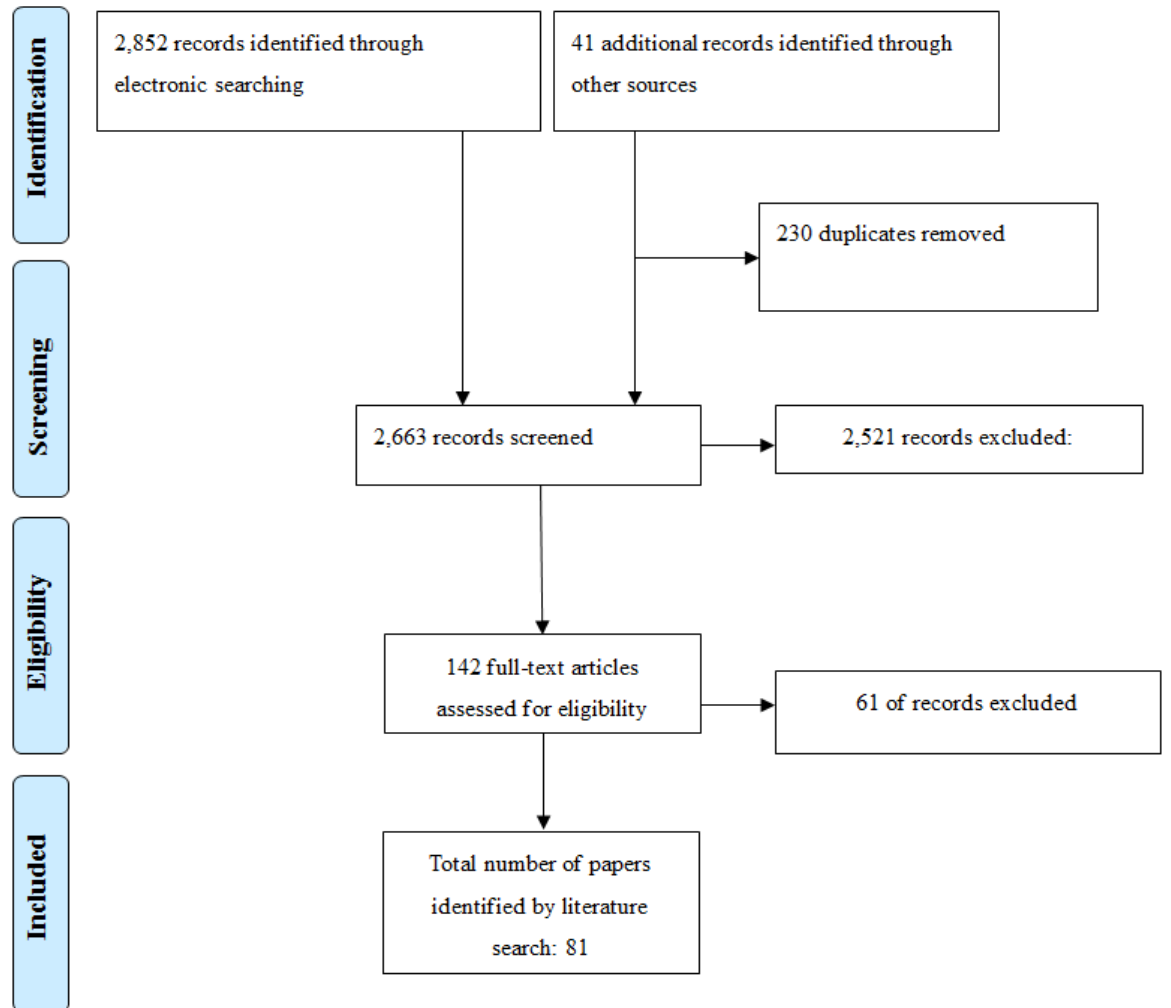

Figure 1. Flow chart of literature search and study inclusion 


\subsection{Measures of risk, positive adaptation and protective factors}

Table 1 demonstrates the variability of measures used to assess risk. The majority of studies employed SES and/or ethnicity as a demonstration of risk or adversity. A wide variety of indexes were utilised to demonstrate the distal risk of low SES including: maternal education, family composition, poverty status, and exposure to community violence. Academic factors were frequently employed as demonstration of risk, including dropping out of high school (Wayman, 2002), having low confidence in graduating (Catterall, 1998), low academic achievement at baseline (Langenkamp, 2010) and low school commitment (Li, Martin, Armstrong, \& Walker, 2011).

The majority of researchers used some form of academic assessment as a demonstration of positive adaptation (e.g. Grade Point Average). In some cases, positive adaptation was indicated using academic achievement, however provided no more information regarding a specific test. In many cases, mathematics and reading scores were used as a measure of total academic achievement (Ladd, Valrie, \& Walcott, 2014; Obradovic et al., 2009). A minority of articles incorporated cognitive aspects of academic achievement, for example, 'self-efficacy in academic domains' (Plunkett, Henry, Houltberg, Sands, \& Abarca-Mortensen, 2008) or academic aspirations (Braddock, Royster, Winfield, \& Hawkins, 1991). Similarly, several studies utilised behavioural assessment of positive adaption by assessing attendance at school (Crosnoe \& Elder, 2004), number of suspensions (Connell, Spencer, \& Aber, 1994), and completed homework (Crosnoe \& Elder, 2004).

Table 1

Indicators of risk, positive adaptation and protective factors in academic resilience research

\begin{tabular}{|c|c|c|c|c|}
\hline First Author (Year) & Assessment of Risk & $\begin{array}{c}\text { Assessment of Positive } \\
\text { Adaptation }\end{array}$ & $\begin{array}{c}\text { Assessment of Protective } \\
\text { Factors }\end{array}$ & $\begin{array}{l}\text { Statistical Analysis } \\
\text { Employed }\end{array}$ \\
\hline Abel (2013) & $\begin{array}{l}\text { Ethnicity, Perceived } \\
\text { discrimination }\end{array}$ & $\begin{array}{l}\text { Grade Point Average } \\
\text { (GPA) }\end{array}$ & $\begin{array}{l}\text { Trait emotional intelligence, } \\
\text { demographic variables }\end{array}$ & $\begin{array}{l}\text { Multiple regression } \\
\text { (mediation analysis) }\end{array}$ \\
\hline Alfaro (2009) & $\begin{array}{l}\text { Ethnicity, Perceived } \\
\text { discrimination }\end{array}$ & GPA & $\begin{array}{l}\text { Academic motivation, English } \\
\text { proficiency, Gender }\end{array}$ & $\begin{array}{l}\text { Structural equation } \\
\text { modelling (mediation) }\end{array}$ \\
\hline Boon (2011) & $\begin{array}{l}\text { Baseline academic } \\
\text { achievement }\end{array}$ & $\begin{array}{l}\text { Follow up academic } \\
\text { achievement }\end{array}$ & $\begin{array}{l}\text { Challenging behaviour, SES, } \\
\text { Gender }\end{array}$ & $\begin{array}{l}\text { Path analysis } \\
\text { (mediation) }\end{array}$ \\
\hline Borman (2004) & Low SES & $\begin{array}{l}\text { Mathematics score } \\
\text { (higher than predicted) }\end{array}$ & $\begin{array}{l}\text { Ethnicity, Individual } \\
\text { characteristics, Peer group, } \\
\text { Effective school variables, } \\
\text { School supportiveness }\end{array}$ & MANOVA \\
\hline Braddock (1991) & Ethnicity & $\begin{array}{l}\text { Academic aspirations, } \\
\text { Peer status, Academic } \\
\text { investments }\end{array}$ & Athletic participation & $\begin{array}{l}\text { Multiple regression } \\
\text { (mediation analysis) }\end{array}$ \\
\hline Cappella (2001) & $\begin{array}{l}\text { Baseline academic } \\
\text { achievement }\end{array}$ & $\begin{array}{l}\text { Follow up academic } \\
\text { achievement }\end{array}$ & $\begin{array}{l}\text { Demographics variables, } \\
\text { Psychological factors, } \\
\text { Behavioural factors, School } \\
\text { factors }\end{array}$ & $\begin{array}{l}\text { Multiple regression } \\
\text { (mediation analysis) }\end{array}$ \\
\hline Catterall (1998) & $\begin{array}{l}\text { Low confidence in } \\
\text { graduating }\end{array}$ & Academic test score & $\begin{array}{l}\text { Family background, Family } \\
\text { academic support, Engagement } \\
\text { in extra-curricular activities, } \\
\text { Teacher responsiveness, } \\
\text { Student attitude towards } \\
\text { motivation }\end{array}$ & $\begin{array}{l}\text { Multiple regression } \\
\text { (mediation analysis) }\end{array}$ \\
\hline Coohey (2010) & Child maltreatment & $\begin{array}{l}\text { Mathematics and } \\
\text { reading test scores }\end{array}$ & $\begin{array}{l}\text { Adaptive behaviour, School } \\
\text { engagement, Behaviour } \\
\text { problems, Relationship with } \\
\text { peers }\end{array}$ & $\begin{array}{l}\text { Multivariate } \\
\text { longitudinal analysis }\end{array}$ \\
\hline Connell (1994) & Gender, Low SES & $\begin{array}{l}\text { GPA, Attendance, } \\
\text { Suspensions (low) }\end{array}$ & $\begin{array}{l}\text { Self-esteem, Perceived } \\
\text { relatedness to self, Perceived } \\
\text { relatedness to other students, } \\
\text { Behavioural engagement }\end{array}$ & Correlation \\
\hline Crosnoe (2004) & $\begin{array}{l}\text { Parent-child emotional } \\
\text { distance }\end{array}$ & $\begin{array}{l}\text { Academic grades, } \\
\text { Completed homework, } \\
\text { Attendance }\end{array}$ & $\begin{array}{l}\text { Parent involvement in } \\
\text { education, Student academic } \\
\text { orientation. }\end{array}$ & $\begin{array}{l}\text { Structural modelling } \\
\text { (tested interactions) }\end{array}$ \\
\hline Elias (2008) & Ethnicity, Low SES & $\begin{array}{l}\text { Reading and } \\
\text { mathematics score }\end{array}$ & $\begin{array}{l}\text { Perceived social support, } \\
\text { Social-emotional competence }\end{array}$ & $\begin{array}{l}\text { Structural equation } \\
\text { modelling (mediation) }\end{array}$ \\
\hline
\end{tabular}


Tudor, K. E., \& Spray, C. M.

Table 1 ...continued

\begin{tabular}{|c|c|c|c|c|}
\hline First Author (Year) & Assessment of Risk & $\begin{array}{l}\text { Assessment of Positive } \\
\text { Adaptation }\end{array}$ & $\begin{array}{c}\text { Assessment of Protective } \\
\text { Factors }\end{array}$ & $\begin{array}{l}\text { Statistical Analysis } \\
\text { Employed }\end{array}$ \\
\hline Fantuzzo (2012) & $\begin{array}{l}\text { Cumulative risk score: } \\
\text { Poverty, Child } \\
\text { maltreatment, Mother } \\
\text { education, } \\
\text { Homelessness, } \\
\text { Inadequate pre-natal } \\
\text { care, Lead exposure }\end{array}$ & $\begin{array}{l}\text { Reading and } \\
\text { mathematics score }\end{array}$ & $\begin{array}{l}\text { Academic engagement, } \\
\text { Attendance }\end{array}$ & $\begin{array}{l}\text { Linear regression } \\
\text { (mediation) }\end{array}$ \\
\hline Farmer (2005) & Ethnicity, Low SES & $\begin{array}{l}\text { Academic, behavioural } \\
\text { and social } \\
\text { characteristics }\end{array}$ & $\begin{array}{l}\text { Behavioural and emotional } \\
\text { strengths }\end{array}$ & $\mathrm{t}$ tests \\
\hline Ferrera (2015) & $\begin{array}{l}\text { Bottom } 1 / 4 \text { of } \\
\text { economic, social and } \\
\text { cultural status }\end{array}$ & $\begin{array}{l}\text { Mathematics score } \\
\text { (top } 1 / 4 \text { nationally) }\end{array}$ & $\begin{array}{l}\text { School variables (e.g. } \\
\text { disruptions in class, class size), } \\
\text { Individual variables (e.g. } \\
\text { attention in mathematics) }\end{array}$ & Logistic regression \\
\hline Finn (1997) & Ethnicity, Low SES & $\begin{array}{l}\text { GPA, Standardised } \\
\text { academic tests, } \\
\text { Graduating on time }\end{array}$ & $\begin{array}{l}\text { Self-esteem, Locus of control, } \\
\text { Engagement }\end{array}$ & MANOVA \\
\hline Geoke-Morey (2012) & Low SES & $\begin{array}{l}\text { Expected academic } \\
\text { attainment }\end{array}$ & $\begin{array}{l}\text { Community, family, parenting } \\
\text { variables }\end{array}$ & $\begin{array}{l}\text { Multiple regression } \\
\text { (no mediation) }\end{array}$ \\
\hline Ghazarian (2010) & Inter-parental conflict & End of year grades & $\begin{array}{l}\text { Youth perceived threat, Youth } \\
\text { self-blame, Maternal } \\
\text { acceptance and monitoring } \\
\text { knowledge }\end{array}$ & $\begin{array}{l}\text { Structural equation } \\
\text { modelling (mediation } \\
\text { and moderation) }\end{array}$ \\
\hline Gonzalez (1997) & Ethnicity & $\begin{array}{l}\text { Academic grades } \\
\text { (mostly As = resilient, } \\
\text { mostly Ds =not } \\
\text { resilient) }\end{array}$ & $\begin{array}{l}\text { Supportive academic } \\
\text { environment, Sense of } \\
\text { belonging in school, Cultural } \\
\text { loyalty }\end{array}$ & $\begin{array}{l}\text { ANOVA, Stepwise } \\
\text { regression \& } \\
\text { Discriminant analysis }\end{array}$ \\
\hline Gordon (1996) & Ethnicity, Low SES & GPA & $\begin{array}{l}\text { Stress, Academic self-concept, } \\
\text { Personality agency beliefs }\end{array}$ & ANOVA \\
\hline Gutman (2002) & $\begin{array}{l}\text { Ethnicity, Household } \\
\text { factors (e.g. maternal } \\
\text { education, depression) }\end{array}$ & $\begin{array}{l}\text { GPA, Attendance, } \\
\text { Mathematics score. }\end{array}$ & $\begin{array}{l}\text { Family factors, Social support } \\
\text { factors }\end{array}$ & $\begin{array}{l}\text { Hierarchical } \\
\text { regression }\end{array}$ \\
\hline Hampton (2016) & Ethnicity, Gender & Academic grades & $\begin{array}{l}\text { Self-respect, English } \\
\text { proficiency, Goal setting } \\
\text { ability, Self-motivation, } \\
\text { Time-management, } \\
\text { Consequence awareness }\end{array}$ & $\begin{array}{l}\text { Linear regression (no } \\
\text { mediation analysis) }\end{array}$ \\
\hline Hawkins (2005) & Ethnicity, SES. & $\begin{array}{l}\text { Educational } \\
\text { aspirations, Peer } \\
\text { status, Academic } \\
\text { investment }\end{array}$ & Athletic participation & $\begin{array}{l}\text { Multiple regression } \\
\text { (mediation analysis) }\end{array}$ \\
\hline Huang (1996) & Ethnicity, Low SES & $\begin{array}{l}\text { Math achievement (top } \\
25 \%=\text { resilient, } \\
\text { bottom } 25 \%=\text { not } \\
\text { resilient) }\end{array}$ & $\begin{array}{l}\text { Motivation, Involvement in } \\
\text { class, Affiliation to others in } \\
\text { class, Learning environment, } \\
\text { Satisfaction, Parent } \\
\text { involvement. }\end{array}$ & ANOVA \\
\hline Irvin (2012) & Ethnicity, Low SES & Academic test score & $\begin{array}{l}\text { Interpersonal competence, } \\
\text { Behavioural engagement, } \\
\text { Psychological engagement, } \\
\text { Aggression. }\end{array}$ & Cluster analysis \\
\hline Kanevsky (2008) & $\begin{array}{l}\text { English learning } \\
\text { student, Low SES }\end{array}$ & Academic test score & $\begin{array}{l}\text { School related psychosocial } \\
\text { variables }\end{array}$ & ANOVA \\
\hline Kwok (2006) & $\begin{array}{l}\text { Baseline academic } \\
\text { achievement, Low } \\
\text { SES }\end{array}$ & $\begin{array}{l}\text { Follow up academic } \\
\text { achievement }\end{array}$ & $\begin{array}{l}\text { Ego resiliency, Agreeableness, } \\
\text { Aggression and hyperactivity, } \\
\text { Cognitive ability, IQ test, } \\
\text { Higher SES }\end{array}$ & Correlation \\
\hline Ladd (2014) & Sickle cell disease & $\begin{array}{l}\text { Math and reading test } \\
\text { score }\end{array}$ & $\begin{array}{l}\text { Family functioning / } \\
\text { environment }\end{array}$ & $\begin{array}{l}\text { Logistic regression } \\
\text { (moderation) }\end{array}$ \\
\hline La Foret (2000) & Ethnicity & $\begin{array}{l}\text { Reading ability, } \\
\text { Verbal ability, GPA }\end{array}$ & $\begin{array}{l}\text { Family factor (e.g. parent } \\
\text { involvement), School } \\
\text { behaviour (e.g. engagement), } \\
\text { Peer relations, Self-concept }\end{array}$ & Correlation \\
\hline Langenkamp (2010) & $\begin{array}{l}\text { Baseline math } \\
\text { achievement (low) }\end{array}$ & $\begin{array}{l}\text { Improved follow up } \\
\text { math score }\end{array}$ & $\begin{array}{l}\text { Social ties pre transition to high } \\
\text { school, Life disruptions } \\
\text { between transitions, School } \\
\text { district }\end{array}$ & Logistic regression \\
\hline Li (2012) & $\begin{array}{l}\text { Poor parental } \\
\text { management, Low } \\
\text { school commitment }\end{array}$ & $\begin{array}{l}\text { GPA (Chinese, } \\
\text { Mathematics, English) }\end{array}$ & $\begin{array}{l}\text { Low truancy, low substance } \\
\text { use, low antisocial behaviour }\end{array}$ & $\begin{array}{l}\text { Hierarchical } \\
\text { regression (no } \\
\text { mediation). } \\
\text { MANOVA }\end{array}$ \\
\hline
\end{tabular}


Table 1 ...continued

\begin{tabular}{|c|c|c|c|c|}
\hline First Author (Year) & Assessment of Risk & $\begin{array}{c}\text { Assessment of Positive } \\
\text { Adaptation }\end{array}$ & $\begin{array}{c}\text { Assessment of Protective } \\
\text { Factors }\end{array}$ & $\begin{array}{l}\text { Statistical Analysis } \\
\text { Employed }\end{array}$ \\
\hline Obradovic (2009) & $\begin{array}{l}\text { Homeless/ highly } \\
\text { mobile, Poverty }\end{array}$ & $\begin{array}{l}\text { Reading and } \\
\text { mathematics score }\end{array}$ & $\begin{array}{l}\text { Demographic and enrolment } \\
\text { variables (e.g. sex, ethnicity) }\end{array}$ & Linear Mixed Models \\
\hline Overstreet (1999) & $\begin{array}{l}\text { Low SES, Exposure to } \\
\text { community violence }\end{array}$ & GPA & $\begin{array}{l}\text { Family environment, Emotional } \\
\text { distress, Depression }\end{array}$ & $\begin{array}{l}\text { Regression analysis } \\
\text { (moderation) }\end{array}$ \\
\hline Peck (2008) & $\begin{array}{l}\text { Ethnicity, Gender, } \\
\text { Low SES }\end{array}$ & Academic test scores & $\begin{array}{l}\text { Self-theories (e.g. perceived } \\
\text { academic ability), World theory } \\
\text { (e.g. positive family } \\
\text { environment), Activity } \\
\text { involvement }\end{array}$ & $\begin{array}{l}\text { Cluster analysis \& } \\
\text { logistic regression }\end{array}$ \\
\hline Perez (2009) & $\begin{array}{l}\text { Ethnicity, } \\
\text { Employment during } \\
\text { high school, Parent } \\
\text { education, Family size }\end{array}$ & GPA & $\begin{array}{l}\text { Personal protective factors (e.g. } \\
\text { valuing school, distress score), } \\
\text { Environmental protective } \\
\text { factors (e.g. family, peers } \\
\text { valuing of school) }\end{array}$ & $\begin{array}{l}\text { Incremental regression } \\
\text { analysis \& cluster } \\
\text { analysis }\end{array}$ \\
\hline Plunkett (2008) & Ethnicity & $\begin{array}{l}\text { Self-efficacy beliefs } \\
\text { for academic learning }\end{array}$ & $\begin{array}{l}\text { Academic support from } \\
\text { significant others }\end{array}$ & MANOVA \\
\hline Raskaukas (2015) & Peer victimisation & GPA & Self-efficacy, Self-esteem & $\begin{array}{l}\text { Hierarchical } \\
\text { regression (mediation } \\
\text { and moderation) }\end{array}$ \\
\hline Reynolds (1998) & Ethnicity, Low SES & $\begin{array}{l}\text { Teacher rating of } \\
\text { classroom adjustment, } \\
\text { Reading and } \\
\text { mathematics scores } \\
\text { (above national } \\
\text { average), Not } \\
\text { repeating grade. }\end{array}$ & $\begin{array}{l}\text { Previous academic } \\
\text { achievement, Perceived } \\
\text { competence, Parent academic } \\
\text { participation, classroom } \\
\text { adjustment }\end{array}$ & Logistic regression \\
\hline Samel (2011) & Low SES & Graduated on time & Classroom environment & Not stated \\
\hline Schelbe (2010) & Maltreated children & Above average grades & Emotional dysregulation & $\begin{array}{l}\text { Linear regression (no } \\
\text { mediation analysis) }\end{array}$ \\
\hline Sharkey (2008) & Low SES & Academic test scores & $\begin{array}{l}\text { Inter-parental conflict, Youth } \\
\text { self-blame, Youth perceived } \\
\text { threat, Maternal acceptance, } \\
\text { Maternal monitoring }\end{array}$ & $\begin{array}{l}\text { Structural equation } \\
\text { modelling (mediation } \\
\text { and moderation) }\end{array}$ \\
\hline Shumow (1999) & Low SES & GPA & $\begin{array}{l}\text { Individual factors (e.g. social } \\
\text { problem solving skills, } \\
\text { academic self-competence), } \\
\text { Family factors (e.g. emotional } \\
\text { support, parent academic } \\
\text { involvement) }\end{array}$ & $\begin{array}{l}\text { Stepwise regression } \\
\text { (no mediation) }\end{array}$ \\
\hline Spencer (1993) & Ethnicity, Low SES & $\begin{array}{l}\text { Academic } \\
\text { achievement (national } \\
\text { achievement percentile } \\
\text { ranking) }\end{array}$ & $\begin{array}{l}\text { Family support, Life } \\
\text { dissatisfaction, Depression, } \\
\text { Self-efficacy }\end{array}$ & $\begin{array}{l}\text { Multiple regression } \\
\text { (no mediation) }\end{array}$ \\
\hline Von Secker (2004) & $\begin{array}{l}\text { Ethnicity, Low SES, } \\
\text { Gender }\end{array}$ & $\begin{array}{l}\text { Science achievement } \\
\text { (standardised test } \\
\text { scores) }\end{array}$ & $\begin{array}{l}\text { Parent education, Home } \\
\text { environment (e.g. reading } \\
\text { material) Attitude towards } \\
\text { science }\end{array}$ & $\begin{array}{l}\text { Hierarchical linear } \\
\text { modelling }\end{array}$ \\
\hline Waxman (1997) & Ethnicity, Low SES & $\begin{array}{l}\text { Mathematics } \\
\text { achievement (top 25\% } \\
=\text { resilient) }\end{array}$ & $\begin{array}{l}\text { Achievement motivation, } \\
\text { Academic self-concept, } \\
\text { Classroom environment, } \\
\text { Satisfaction in Maths, Parent } \\
\text { academic involvement }\end{array}$ & $\begin{array}{l}\text { Chi-square test \& } \\
\text { MANOVA }\end{array}$ \\
\hline Wayman (2002) & High school drop out & $\begin{array}{l}\text { Completed GED, } \\
\text { Returned to high } \\
\text { school }\end{array}$ & $\begin{array}{l}\text { SES, Family and peer factors } \\
\text { (support), School factors (e.g. } \\
\text { extra-curricular activity), Age } \\
\text { at dropout, Parent status }\end{array}$ & Logistic regression \\
\hline Woolley (2007) & $\begin{array}{l}\text { Threat to safety/ } \\
\text { security, High risk } \\
\text { peer affiliations, } \\
\text { Social stressors }\end{array}$ & Academic success & $\begin{array}{l}\text { Supportive / caring adults in the } \\
\text { home }\end{array}$ & $\begin{array}{l}\text { Regression analysis } \\
\text { (mediation) }\end{array}$ \\
\hline
\end{tabular}


Table 2

Measurement Scales Utilised in Academic Resilience Research

\begin{tabular}{|c|c|c|}
\hline $\begin{array}{l}\text { First Author } \\
\text { (Year) }\end{array}$ & Risk Status & Measure of Resilience \\
\hline $\begin{array}{l}\text { Banatao (2011); } \\
\text { Hanson (2013); } \\
\text { Jowkar (2011) }\end{array}$ & Low SES & $\begin{array}{l}\text { Resilience and Youth Development Module (RYDM). } 58 \text { items measuring internal student assets } \\
\text { linked to positive developmental outcomes: a) school assets (caring relationships high expectations, } \\
\text { meaningful participation) b) home assets (caring relationships, high expectations, participation at } \\
\text { home) c) community assets (caring relationships, high expectations, meaningful participation) d) } \\
\text { Peer assets (caring relationships, pro-social peers) Internal resilient assets ( } 3 \text { items each): } \\
\text { co-operation, self-efficacy, empathy, problem solving, self-awareness. }\end{array}$ \\
\hline Burger (2006) & Not stated & $\begin{array}{l}\text { Subscales of the Student-Orientation-to-School Questionnaire: External resilience, 'perceived } \\
\text { ability to cope and adapt successfully in the face of challenges': } 11 \text { items, e.g. "I pull through } \\
\text { when things are difficult"). Internal resilience, 'perceived ability to resist anxiety and maintain } \\
\text { internal emotional and mental balance': } 4 \text { items, e.g. "making mistakes bugs me". Responses on a } 5 \\
\text { point scale (ranging from strongly agree to strongly disagree). }\end{array}$ \\
\hline Martin (2006) & Not stated & $\begin{array}{l}\text { Academic Resilience (6 items: "I believe I am mentally tough when it comes to exams"; "I don't let } \\
\text { study stress get on top of me", "I'm good at bouncing back from a poor mark in my schoolwork", "I } \\
\text { think I'm good at dealing with schoolwork pressures", "I don't let a bad mark affect my confidence", } \\
\text { "I'm good at dealing with setbacks at school e.g. bad mark, negative feedback). }\end{array}$ \\
\hline Martin (2008) & Not stated & $\begin{array}{l}\text { Academic Buoyancy Scale (ABS). } 4 \text { items: "I'm good at dealing with setbacks (e.g. bad mark, } \\
\text { negative feedback on my work", "I don't let study stress get on top of me" "I think I am good at } \\
\text { dealing with schoolwork pressures", "I don't let a bad mark affect my confidence" }\end{array}$ \\
\hline Martin (2013) & $\begin{array}{l}\text { Previous } \\
\text { academic } \\
\text { adversity }\end{array}$ & $\begin{array}{l}\text { Academic Risk and Resilience Scale (ARRS): Students indicated 'yes' or 'no' to ten major } \\
\text { academic adversity items (e.g. suspension, skipped a grade). Those who selected 'yes' to at least one } \\
\text { academic adversity then asked to answer for items on a } 7 \text { point scale ( } 1=\text { strongly disagree, } 7= \\
\text { strongly disagree): "I don't let these types of difficulties get on top of me", "I think I'm good at } \\
\text { dealing with these types of pressures", "I don't let these types of difficulties affect my confidence", } \\
\text { "I'm good at overcoming these types of setbacks". }\end{array}$ \\
\hline Phan (2016) & Not stated & $\begin{array}{l}\text { Vigour (i.e. persistence and resilience), subscale of the Engagement scale (Scaufeli et al., 2002). } 6 \\
\text { items, } 7 \text { point scale, e.g. "As far as my studies in maths are concerned I always persevere, even } \\
\text { when things do not go well" }\end{array}$ \\
\hline Esteban (2104) & $\begin{array}{l}\text { Immigrant } \\
\text { status }\end{array}$ & Resilience Scale (Saavedra \& Villalta, 2008), evaluates 12 resources that students may use. \\
\hline Sarwar (2010) & Not stated & $\begin{array}{l}\text { Resilience Scale developed for the study, } 6 \text { dimensions: } 1 \text {. Personal competence 2. Social } \\
\text { Competence 3. Family competence } 4 \text {. Personal Structure 5. Social Support } 6 \text {. Total Resilience }\end{array}$ \\
\hline Skinner (2013) & Not stated & $\begin{array}{l}\text { Resilience Measure: } 3 \text { components of resilience. } 1 \text {. Engagement versus disaffection. A) } \\
\text { Behavioural engagement ( } 5 \text { items }) \text {; b) behavioural disaffection ( } 5 \text { items); emotional engagement ( } 6 \\
\text { items); emotional disaffection ( } 10 \text { items). } 2 \text {. Emotional Reactivity (extent to which student reacts } \\
\text { negatively when they run into academic problems; } 11 \text { items). } 3 \text {. Reengagement in face of academic } \\
\text { challenge, tapping into mastery reactions ( } 4 \text { items) and giving up ( } 5 \text { items) }\end{array}$ \\
\hline Thornton (2006) & Ethnicity & $\begin{array}{l}\text { Resiliency Belief System Instrument (Jew et al., 1999): } 50 \text { items, } 5 \text { subscales: } 1 \text {. The Active } \\
\text { Optimism Subscale ( } 17 \text { items) 2. Passive Optimism Subscale (17 items) 3. Active Belief in Others } \\
\text { Subscale (10 items) 4. Passive Belief in Others Subscale (6 items) 5. Total Scores. }\end{array}$ \\
\hline
\end{tabular}

\subsection{Statistical analysis}

Variable-focused approaches - Most of the studies in the current review employed some form of mediation or moderation analysis to assess the influence of specific protective (or vulnerability) factors on the relationship between risk and positive adaptation. Table 1 demonstrates the form of statistical analysis used to examine the role of protective factors in the relationship between risk and adaptation. Many used multiple regression to test the mediating influence of specific protective factors on educational outcomes. For example, Braddock et al. (1991) tested the role of athletic activity in mediating the relationship between ethnicity and academic aspirations. In some cases, correlation analysis was used to assess relationships. For example, LaForrett (2000) investigated the role of family factors, self-concept and school behavior on academic achievement in at-risk students from an ethnic-minority background. Using correlation analysis fails to identify the specific impact of a protective factor on educational outcomes. Moreover, the majority of studies used a cross-sectional design with data collected at one time point.

Person-focused approaches - Where a person-focussed approach was employed, participants were grouped as 'resilient' or 'not resilient' based on their positive adaptation at school. For example, in studies by Huang and Waxman (1996) and Waxman (1997), participants in the top 25\% for mathematics achievement were grouped as resilient, while the bottom $25 \%$ were grouped as not resilient. Similarly, Wayman (2002) grouped resilient and 
non-resilient as those who either did or did not complete their Graduate Education Diploma (GED) following dropping out of high school. Many other studies employed statistical analysis which involved comparison of groups however did not state the 'cut off' point for a resilient or a non-resilient student. For studies employing a person-focused approach, cluster analysis, or comparison of groups using analysis of variance was most frequently utilized. Differences in levels of a number of protective factors were then observed. For example, Irvin (2012) utilized cluster analysis, to determine whether psychological and behavioral engagement served as a protective factor in profiles of resilient and non-resilient students. Similarly, Peck et al. (2008) investigated the role of positive family environment and perceived academic ability in high achieving and low achieving students using logistic regression and cluster analysis.

\subsection{Measurement scales}

Table 2 demonstrates the measurement scales utilised to assess academic resilience. Most scales incorporated a variety of protective factors that are linked to positive adaptation, with each item indicating one protective factor. The Resilience and Youth Development Module (RYDM; Hanson \& Kim, 2007) consists of 58 items measuring internal student assets linked to adaptive outcomes, including; school assets, home assets, community assets, peer assets and internal resilient assets. Similarly, the academic resilience scale developed for Sarwar's (2010) study incorporates five subscales reflecting different assets: personal competence, social competence, family competence, personal structure and family support. Moreover, Skinner's (2013) resilience measure includes three components of resilience; engagement, emotional reactivity and reengagement (described as similar to the concept of 'academic buoyancy'). Finally, Phan (2016) measured academic vigour (i.e. combination of persistence and resilience), using a subscale of the validated scale of Academic Engagement (Schaufeli, Salanova, Gonzalez-Roma, \& Bakker, 2002).

In contrast, a small number of scales measured academic resilience using a univariate scale assessing students' responses to academic adversities. For example, the Academic Resilience scale (Martin \& Marsh, 2006) consisted of six items reflecting students' cognitive response to setbacks at school (e.g. "I am mentally tough when it comes to exams"). This scale was subsequently amended, with the removal of two items ("I am mentally tough when it comes to exams" and "I'm good at bouncing back from a poor mark in my schoolwork", to form the Academic Buoyancy Scale (Martin \& Marsh, 2008). Both scales were developed to assess how students responded to more minor academic adversities. Finally, Martin (2013) developed the Academic Risk and Resilience Scale (ARRS; Martin, 2013) to assess how students respond to more severe academic adversities. As described in Table 2, this scale requires participants to answer 'yes' or 'no' to ten significant academic adversities (e.g. suspension) then respond to an amended version of the ABS ("I don't let these types of difficulties get on top of me", "I think I'm good at dealing with these types of pressures").

\section{Discussion}

Recent years have seen an influx in government funding for the implementation of resilience programmes in schools (Schofield \& Bates, 2016). Such interventions, however, have been implemented with little understanding of the desired outcome (i.e. resilience), and no specific outcome measures to evaluate such programmes. The aim of the current study therefore, was to provide an overview of methodologies employed to assess academic resilience. Specifically, we aimed to report the indicators of risk and positive adaptation used to infer students' academic resilience and the statistical analyses employed to determine the role of protective factors. Finally, we aimed to identify studies that employed measurement scales to assess academic resilience.

The review identified a number of scales utilised to measure academic resilience. Furthermore, there was heterogeneity in the indicators of risk and positive adaptation that were utilised to reflect academic resilience. This inconsistency reflects ongoing debate regarding the conceptualisation of academic resilience and the difficulties in developing an operational definition of the construct. Similar definitional and conceptual issues have been highlighted in the psychological resilience literature. The following discussion therefore uses the 
Tudor, K. E., \& Spray, C. M.

lessons learned from the traditional literature to make recommendations for the development of a valid and reliable measure of academic resilience.

\subsection{Assessing risk}

It has been proposed in the psychological resilience literature that measures should assess three defining components: risk, positive adaptation and protective factors (Sarkar \& Fletcher, 2013). Turning first to risk, the current review highlights the heterogeneity of factors used to represent risk. Most frequently utilised to represent academic risk was socio-economic status, which was assessed in many different ways across studies (e.g. maternal education, exposure to community violence). While it is important and exciting to understand students from a variety of adverse circumstances, the diversity of risks presents a problem for comparing and interpreting results. Although the literature is very clear that socio-demographic factors predict negative academic outcomes, using a single indicator, for example ethnicity, makes the assumption that all students within this demographic are at equal risk of poor academic outcomes. This approach therefore encompasses students from low SES backgrounds who are academically gifted, and similarly excludes students from high SES backgrounds who show significant trouble in the face of academic adversity. Thus, educational scholars should seek to use academic indicators of risk, for example, 'low confidence in graduating' or 'low baseline academic achievement'.

In empirical studies of psychological resilience, one approach to measuring risk, or adversity, is the use of checklists of negative life events (Luthar \& Cushing, 1999). Measurement scales such as, the Life Events Checklist (Work, Cohen, Parker, \& Wyman, 1990) and the Daily Hassles Scale (Kanner, Coyne, Schaefer, \& Lazarus, 1981) have been used to measure major and minor life events to gain a complete picture of risk. The academic resilience literature would benefit from the development of a checklist of events that are associated with academic disengagement and substandard academic motivation. Martin (2013) has approached this method of assessment with the development of the Academic Risk and Resilience Scale, which incorporates major academic events associated with disengagement from school (e.g. 'suspended from school', 'did not hand in most assignments', 'major illness affecting schoolwork'). This approach, paired with an appropriate measure of positive adaptation is a step in the right direction in assessing academic resilience. It is important, however, to be transparent regarding item generation, thus future development of a checklist for academic adversities should be generated from qualitative research with teachers, students and parents, exploring the chronic and acute events that impact students' academic outcomes.

One concern for researchers considering the option of developing a checklist for academic risk relates to the need to differentiate between chronic circumstances and acute events, as these are associated with different outcomes (Masten, Neeman, \& Adenas, 1994). Academic resilience researchers therefore should take into account the recording of the frequency of events student's experience. Moreover, another consideration with this type of assessment is the potential for measurement confounds. For example, within the psychological resilience literature, Luthar and Cushing (1999) suggest that the inclusion of controllable adversities when measuring risk can inflate the relationship between risk and adaptation. Therefore, scales aiming to assess adversities that pose a risk to poor academic achievement should exclude those that are clearly controllable by the student (e.g. 'suspended from school'). For some cases there may be ambiguity, therefore the most rigorous approach would be to generate events through qualitative enquiry and request a panel of experts to rate the events in regards to their controllability (Luthar \& Cushing, 1999).

As noted, educational psychologists propose that academic resilience does not address the majority of students who face less extreme academic adversities associated with everyday school life (e.g. patches of poor performance, pressures of competing deadlines). Thus, academic buoyancy refers to students' ability to "successfully deal with academic setbacks and challenges that are typical of the ordinary course of school life" (Martin \& Marsh, 2008, p.54). Buoyancy therefore reflects an 'everyday resilience' that is more relevant for the majority of students who deal with the challenges of school. Martin and colleagues use a measurement scale to 
Approaches to measuring academic resilience: A systematic review

assess students' responses to more minor adversities, which will be discussed in more detail below. However we suggest assessing buoyancy using the same approach as resilience, specifically, measuring academic risk, positive adaptation and protective factors independently. Using this approach, scholars should strive to measure the everyday difficulties and challenges associated with everyday school life that may impact on students educational outcomes.

Research within the stress exposure literature has suggested that everyday academic events (e.g. problems relating with teachers, being involved in too many extracurricular activities or being disrupted by peers) has an influence on the students wellbeing and academic performance (Escobar et al., 2013). As a result, Escobar and colleagues developed a measure of children's daily stressors (Trianes, Blanca, Fernandez-Baena, Escobar, \& Maldonado, 2011), comprising three first-order factors related to family, health and school. School related items include, "I find schoolwork difficult", "the other children pick on me a lot at school". Thus, the academic buoyancy literature may benefit from the development of a scale depicting everyday academic events that students must be resilient against. Again, items should be identified through exploratory, qualitative methodologies, with transparent reporting of item development. Doing so would ensure content validity and that the items in the questionnaire truly represent the construct in the given population (Windle et al., 2011).

\subsection{Assessing positive adaptation}

Educational researchers should strive to measure positive adaptation, in conjunction with risk and protective factors, to gain a complete understanding of academic resilience. Positive adaptation refers to, success at meeting stage salient developmental tasks, or adaptation that is substantially better than that would be expected given the specific risk exposure. Luthar and Cushing (1999) state three approaches to measuring positive adaptation in psychological resilience, one of which is the absence of serious psychopathology. This approach has been utilized in the academic resilience domain, with scholars using the 'absence of academic failure' as an indicator of positive adaptation. Most defined resilience in terms of the single outcome of an academic test score or an average of test scores, most commonly in Mathematics and/ or Reading. In person-focused approaches, scholars define resilient students as those who achieve academic scores within the top $25 \%$ of the national average and non-resilient as those who score in the bottom 25\%. Assessing positive adaptation, and inferring resilient outcomes, in this way provides a limited view of what education is about. Focusing solely on test results overlooks students who may be naturally very intelligent however struggle when it comes to autonomous learning or problem solving (Pianta \& Walsh, 1998). For example, a highly intelligent student may outperform all of his or her peers, yet disengage when they are eventually faced with difficulty. Likewise, a less intelligent student may perform poorly on academic test results, despite being very resilient in the face of such academic challenges. A more appropriate measure of positive adaptation may be students' level of academic engagement, motivation, or aspirations (Braddock et al., 1991; Hawkins \& Mulkey, 2005).

Another broad approach to measuring positive adaptation in the psychological resilience literature is through the development of multi-item measures, scoring on a continuum between adjustment (i.e. competence) and maladjustment. Within the current review, no scholars took this approach to measuring positive adaptation (Luthar \& Cushing, 1999). Educational scholars may take this approach by utilising an existing measure of adjustment relevant to the academic context, for example a measure of academic engagement or motivation (e.g. Engagement and Disaffection Scale; Skinner, \& Wellborn, 1997). Academic engagement is distinguished from academic resilience in that engagement refers to students' enthusiastic and focussed participation in the classroom (i.e. pay attention, display interest and, work hard). In contrast to academic resilience, academic engagement does not encompass a specific academic risk, therefore could represent the positive adaptation component of resilience. If this approach were undertaken however, scholars should take some considerations into account. Firstly, that the indicator of positive adaptation is specific to the risk under scrutiny. This becomes relevant for school-based resilience interventions targeting students that are at-risk for psychological dysfunction, as opposed to academic risk. Such interventions should use psychological indicators of positive adaptation (not academic achievement), while interventions aimed at fostering academic resilience should use academic 
indicators of positive adaptation. Second, scholars should consider the seriousness of the risk under consideration. For example, if a student is exposed to a very serious academic risk (e.g. repeating a grade, learning difficulty) it would be sufficient to justify lower scores on a measurement of competence (Luthar \& Cushing, 1999). In contrast, if a student experiences less taxing academic risks, for example the daily hassles described in the buoyancy literature, a resilient student should demonstrate excellent scores on a measurement of competence.

\subsection{Assessing protective factors}

Protective factors refer to internal attributes or external resources that "modify, ameliorate, or alter a person's response to an environmental hazard" (Rutter, 1985, p. 600). The results of the current review demonstrate the variability in the protective factors that have been investigated in academic resilience research. Factors protecting individuals from academic risk included demographic (e.g. SES), individual (e.g. emotional intelligence), family (e.g. parent involvement in education) and, other social factors (e.g. peer group / school supportiveness). With regards to academic buoyancy, the 5Cs (composure, control, commitment, confidence, and coordination) protected students from the everyday academic pressures they experienced at school. Within the buoyancy literature there is more consistency in the protective factors investigated, however within academic resilience, the heterogeneity of investigated factors make the development of interventions very difficult.

A common concern in the traditional resilience research, also identified in the current review, is the blurred distinction between risk and protective factors. Here, authors should consider the psychometric issues relating to both variable-focused and person-focussed approaches to investigating resilience. In most cases, regression analyses or structural equation modelling were employed to test the mediating and/ or moderating role of protective factors. In some cases, however, linear regression was conducted to test the predictive value of each protective factor on a given academic outcome, with no mediation or moderation analyses. To illustrate, Li et al. (2011) tested linkages between measures of risk (i.e. low school commitment) and protective factors (i.e. low truancy and antisocial behaviour), however did not test the mediating or moderating effect of such factors. This approach does not identify factors that "modify" or "ameliorate" the effect of the risk on positive adaptation. The choice of statistical analysis is important here, to ensure a protective factor is being tested within a model of academic resilience. That is, a protective factor is being tested for how it impacts the relationship between risk and positive outcomes, rather than its direct predictive utility on the outcome. If authors claim to be assessing academic resilience, the appropriate assessment of protective factors is recommended.

This concern was also present in some person-focussed investigations. Using this approach, researchers sought to identify groups of individuals, from the same high risk sample, with good versus poor academic outcomes, and test which factors accounted for the differences in outcomes. To illustrate, Finn and Rock (1987) identified academically resilient students from at-risk groups (i.e. low-SES and ethnic minority students) through the demonstration of grades and school completion. Differences between these resilient and non-resilient students were then identified by comparing groups using analysis of variance. Again, this approach does not identify specific protective factors that function to shield students from the negative effects of academic risk (Masten, 2001).

The majority of the variable-focussed studies in the current review were cross-sectional in design. A recommendation for future research is to examine how adversities and protective factors influence each other over time to predict academic outcomes. It may be that a risk factor, for example academic disengagement may predict the quality of teacher support over time, and also that teacher support predicts academic engagement over time, both contributing to increased academic success. To fully understand the transactional dynamics of individual students and their environment, and how this impacts on positive academic outcomes, scholars should attempt to employ longitudinal designs. Within the psychological resilience literature, Luthar et al. (2000) proposes scholars should obtain measurements on three occasions, with an appropriate distance between time-points to enable the hypothesised protective factors to take effect. In the academic context, it may be 
appropriate to assess protective factors before, during and after a demanding period, for example an exam, to identify the changing relationship between academic risks and outcomes.

A final recommendation for research employing a person-focussed approach may be to consider the comparison of a third group: students with low risk, who have good academic outcomes. Doing so, researchers could better understand if high-risk, resilient students share any specific protective factors with their low-risk, academically competent peers (Masten, 2001). This method of comparing three groups has been used in the traditional resilience literature, which has found that maladaptive groups of children can be discriminated from the two adaptive groups (high and low-risk), however the two adaptive groups cannot not be discriminated from each other. Applying this method to academic resilience may uncover which factors define academic resilience in students.

\subsection{Assessment using measurement scales}

Over the past three decades, the approach of identifying protective factors has made a significant contribution to the development of a number of measures of psychological resilience (see for a review Windle et al., 2011) The most conceptually sound measures are those that comprise a number of items representing characteristics that enable individuals to positively adapt to the risks they face. Within the current review, ten measurement scales were used to measure academic resilience; however there were a number of psychometric issues relating to their use. The first problem relating to measurement scales is face validity. To illustrate, Phan et al. (2016) adapted a scale that was developed and validated to measure burnout in working adults and university students. Similarly, Esteban and Marti (2014) utilized a measure of resilience developed and validated in a non-academic sample of adults to assess academic resilience in students. When scholars make use of existing measures of resilience it is important they make a strong rationale for doing so, and provide details of the original measure development. Similarly, with regards to face validity, some scales may assess phenomenon that are related to resilience, however conceptually distinct. For example, Phan (2014) measured 'vigour', defined as a combination of persistence and resilience, using the subscale of an engagement scale (Scaufeli et al., 2002) consisting of items such as, "as far as my studies in maths are concerned I always persevere, even when things are not going well". While resilience and persistence have some overlapping characteristics, and are often used interchangeably, they are in fact conceptually distinct. It is important that the development of future academic resilience scales are distinguished from any related concepts to avoid confusion for practitioners incorporating resilience in an applied setting.

The second problem regarding measures used identified in the review relates to the limited evidence-base for item selection. For example, the ABS (Martin \& Marsh, 2008) was developed through the amendment of the ARS (Martin \& Marsh, 2006), which involved the removal of two items. However little information is provided regarding the theoretical basis for the selection of the original six items, and the decision to remove two items to create the ABS. Moreover, the items that comprise resilience scales differ based on the authors' conceptualisation of the concept. This is illustrated by Martin and Marsh's $(2006,2008)$ specific focus on 'bouncing back' from academic adversity (e.g. "I think I am good at dealing with schoolwork pressures").

\subsection{Limitations}

The current study undertook an extensive literature search to identify relevant articles; however, it only reports published studies in the English language. It is possible that there are relevant studies published in other languages which were not included in this review. In addition, there were some articles identified in the screening process that were unavailable to the authors. Although an attempt was made by the authors to gain a copy (through contacting corresponding authors), some were not ascertained. Furthermore, the current review did not employ published quality assessment criteria to assess the psychometric rigour of each scale. This was due to the small number of scales identified that were developed and validated for the sole purpose of assessing academic resilience. 


\section{Conclusions}

Academic resilience is receiving more interest in terms of policy practice; however there is not yet a valid and reliable measure to assess the concept. The key recommendations that emerge from the discussion are fourfold. First, measures of academic resilience should incorporate three components: risk, positive adaptation, and protective factors separately. Second, when assessing risk, researchers should use academic indicators of risk, and consider the development of a scale of both stressors and everyday hassles relating to school. Third, researchers should consider alternative indicators of positive adaptation than academic achievement, for example emotional and behavioral engagement at school. Finally, when assessing protective factors, the appropriate statistical analysis should be used to examine how the factor moderates the effect of an academic risk on academic outcomes. Researchers should strive to utilize the lessons learned in psychological resilience literature to establish a reliable and valid measure, and gain a complete picture, of academic resilience.

\section{References}

Abel, N. R. (2013). Trait emotional intelligence, perceived discrimination, and academic achievement among African American and Latina/o high school students: A study of academic resilience (Unpublished doctoral dissertation). Minnesota State University. Mankato, Minnesota.

Afifi, T. O., \& Macmillan, H. L. (2011). Resilience following child maltreatment: a review of protective factors. Canadian Journal of Psychiatry, 56(5), 266-272. https://doi.org/10.1177/070674371105600505

Alfaro, E. C., Taylor, A. J., Gonzales-Backen, M. A., Bamaca, M. Y., \& Zeiders, K. H. (2009). Latino adolescents' academic success: The role of discrimination, academic motivation, and gender. Journal of Adolescence, 32(4), 941-962. http://dx.doi.org/10.1016/j.adolescence.2008.08.007

Alva, S. A. (1991). Academic invulnerability among Mexican-American students: The importance of protective resources and appraisals. Hispanic Journal of Behavioural Sciences, 13(1), 18-34. https://doi.org/10.1177/07399863910131002

Banatao, E. J. (2011). Educational resilience: The relationship between school protective factors and student achievement (Unpublished doctoral dissertation). San Diego State University, California.

Bonanno, G. A. (2004). Loss, trauma, and human resilience: have we underestimated the human capacity to thrive after extremely aversive events? American Psychologist, 59(1), 20-28.

Boon, H. J. (2008). Risk or resilience? What makes a difference? The Australian Educational Researcher, 35(1), 81-102.

Borman, G. D., \& Rachuba, L. T. (2001). Academic success among poor and minority students: an analysis of competing models of school effects. Baltimore, MD: John Hopkins University, Center for Research on the Education of Students Placed at Risk. Retrieved from http://eric.ed.gov/?id=ED451281

Braddock, J. H., Royster, D. A., Winfield, L. F., \& Hawkins, R. (1991). Bouncing back: Sports and academic resilience among African-American males. Education and Urban Society, 24(1), 113-131. https://doi.org/10.1177/0013124591024001009

Burger, J. M., Nadirova, A., \& Keefer, K. V. (2012). Moving beyond achievement data: Development of the Student Orientation to School Questionnaire as a non-cognitive assessment tool. Journal of Psychoeducational Assessment, 30(4), 367-386. http://dx.doi.org/10.1177/0734282912449444

Cappella. E., \& Weinstein, R. S. (2001). Turning around reading achievement: predictors of high school students' academic resilience. Journal of Educational Psychology, 93(4), 758-771. https://doi.org/10.1037/0022-0663.93.4.758

Catterall, J. S. (1998). Risk and resilience in student transitions to high school. American Journal of Education, 106(2), 302-333. https://doi.org/10.1086/444184

Cheung, K., Sit, P., Soh, K., \& Soi-kei Mak, M. (2014). Predicting academic resilience with reading engagement and demographic variables: Comparing Shanghai, Hong Kong, Korea, and Singapore from the PISA perspective. The Asia-Pacific Education Researcher, 23(4), 895-909. https://doi.org/10.1007/s40299-013-0143-4 
Approaches to measuring academic resilience: A systematic review

Collie, R. J., Martin, A. J., Malmberg, L. E., Hall, J., \& Ginns, P. (2015). Academic buoyancy, student's achievement, and the linking role of control: A cross-lagged analysis of high school students. British Journal Educational Psychology, 85(1), 113-130. http://dx.doi.org/10.1111/bjep.12066

Connell, J. P., Spencer, M. B., \& Aber, J. L. (1994). Educational risk and resilience in African-American youth: Context, self, action, and outcomes in school. Child Development, 65(2), 493-506. https://doi.org/10.2307/1131398

Connor, K. M., \& Davidson, J. R. T. (2003). Development of a new resilience scale: The Connor-Davidson resilience Scale (CD-RISC). Depression and Anxiety, 18(2), 76-82. https://doi.org/10.1002/da.10113

Constantine, N., Benard, B., \& Diaz, M. (1999, June). Measuring protective factors and resilience traits in youth: The healthy kids resilience assessment. Paper presented at the Seventh Annual Meeting of the Society for Prevention Research, New Orleans, LA.

Coohey. C. Renner, L. M., Hua, L., Zhang, Y. J., \& Whitney, S. D. (2011). Academic achievement despite child maltreatment. Child Abuse and Neglect, 35(9), 688-699. https://doi.org/10.1016/j.chiabu.2011.05.009

Cooper, C. E., \& Crosnoe, R. (2007). The engagement in schooling of economically disadvantaged parents and children. Youth \& Society, 38(3), 372-391. https://doi.org/10.1177/0044118X06289999

Crosnoe, R., \& Elder, G. H. (2014). Family dynamics, supportive relationships, and educational resilience during adolescence. Journal of Family Issues, 25(5), 571-602. https://doi.org/10.1177/0192513X03258307

Elias, M. J., \& Haynes, N. M. (2008). Social competence, social support, and academic achievement in minority, low-income, urban elementary school children. School Psychology Quarterly, 23(4), 474-495. http://dx.doi..org/10.1037/1045-3830.23.4.474

Escobar, M., Alarcon, R., Rosel, J. F., Fernandez-Baena, F. J., \& Trianes, M. V. (2013). Daily stressors in school-age children: A multi-level approach. School Psychology Quarterly, 28(3), 227-238. https://doi.org/10.1037/spq0000020

Esteban, S., \& Marti, A. S. (2014). Beyond compulsory schooling: Resilience and academic success in immigrant youth. Social and Behavioural Sciences, 132, 19-24. https://doi.org/10.1016/j.sbspro.2014.04.272

Fantuzzo, J., LeBoeuf, W., Rouse, H., \& Chen, C. C. (2012). Academic achievement of African American boys: A city-wide, community-based investigation of risk and resilience. Journal of School Psychology, 50(5), 559-579. https://doi.org/10.1016/jsp.2012.04004

Farmer, T. W., Clemmer, J. T., Leung, M., Goforth, J. B., Thompson, J. H., Keagy, B. S., \& Bloucher, S. (2005). Strength-based assessment of rural African American early adolescents: characteristics of students in high and low groups on the behavioural and emotional rating scale. Journal of Child and Family Studies, 14(1), 57-69. https://doi.org/10.1007/s10826-005-1113-0

Ferrera, J. M. C. (2015). Factors promoting educational attainment in unfavourable socioeconomic conditions. Revista De Education, 370, 172-198. https://doi.org/10.4438/1988-592X-RE-2015-370-302

Finn, J. D., \& Rock, D. A. (1997). Academic success among students at risk for school failure. Journal of Applied Psychology, 82(2), 221-234. https://doi.org/10.1037/0021-9010.82.2.221

Garmezy, N., \& Masten, A. S. (1986). Stress, competence, and resilience. Common frontiers for therapist and psychopathologist. Behaviour Therapy, 17(5), 500-521.

Geoke- Morey, M. C., Taylor, L. K., Merrilees, C. E., Cummings, M., Cairns, E., \& Shirlow, P. (2012). Adolescents' educational outcomes in a social ecology of parenting, family, and community risks in Northern Ireland. School Psychology International, 34(3), 243-256. https://doi.org/10.1177/0143034311435162

Ghazarian, S. R., \& Buehler, C. (2010). Inter-parental conflict and academic achievement: An examination of mediating and moderating factors. Journal of Youth Adolescence, 39(1), 23-35. https://doi.org/10.1007/s10964-008-9360-1

Gillham, J. H., Reivich, K. J., Freres, D. R., Chaplin, T. M., Shatte, A. J., \& Samuels, B. (2007). School-based prevention of depressive symptoms: A randomized controlled study of the effectiveness and specificity of the Penn resiliency program. Journal of Consulting and Clinical Psychology, 75(1), 9-19. https://doi.org/10.1037\%2F0022-006X.75.1.9 
Tudor, K. E., \& Spray, C. M.

Gonzalez, R., \& Padilla., A. M. (1997). The academic resilience of Mexican American high school students. Hispanic Journal of Behavioural Sciences, 19(3), 301-317. https://doi.org/10.1177/07399863970193004

Gordon, K. A. (1996). Resilient Hispanic youths' self-concept and motivational patterns. Hispanic Journal of Behavioural Sciences, 18(1), 63-73. https://doi.org/10.1177/07399863960181007

Gutman, L. M., Sameroff, A. J., \& Eccles, J. S. (2002). The academic achievement of African American students during early adolescence: An examination of multiple risk, promotive and protective factors. American Journal of Community Psychology, 30(3), 367- 399. https://doi.org/0091-0562/02/0600-0367/0

Hampton, F. M. (2016). The seven secrets of successful urban school students: The evidence that continues to grow. Education and Urban Society, 48(5), 423-443. https://doi.org/10.1177/0013124514533990

Hanson, T. L., \& Kim, J.O. (2007). Measuring resilience and youth development: The psychometric properties of the healthy kids survey. Issues \& Answers Report, REL 2007- No. 034). Washington, DC: U.S. Department of Education, Institute of Education Sciences, National Centre for Education Evaluation and Regional Assistance, Regional Educational Laboratory West. Retrieved from http://ies.ed.gov/ncee/edlabs

Hart, A., \& Heaver, B. (2013). Evaluating resilience-based programs for schools using a systematic consultive review. Journal of Child and Youth Development, 1(1), 27-53.

Hart, A., \& Heaver, B. (2015). Resilience interventions: What works? Retrieved from http://www.boingboing.org.uk/wp-content/uploads/2017/02/angie-and-becky-school-based-resilience-a pproaches-dec-12.pdf

Hawkins, R. \& Mulkey, L. M. (2005). Athletic investment and academic resilience in a national sample of African American females and males in the middle grades. Education and Urban Society, 38(1), 62-88. https://doi.org/10.1177/0013124505280025

Huang, S. L., \& Waxman, H. C. (1996). Comparing learning environment of resilient and non-resilient Asian American students. Paper presented at the Annual Meeting of the American Educational Research Association.

Irvin, M. J. (2012). Role of student engagement in the resilience of African American adolescents from low-income rural communities. Psychology in the Schools, 49(2), 176-193. https://doi.org/10.1002/pits.20626

Jew, C. L., Green, K. E., \& Kroger, J. (1999). Development and validation of a measure of resiliency. Measurement and Evaluation in Counselling and Development, 32(2), 75-89.

Jowkar, B., Kohoulat, N., \& Zaken, H. (2011). Family communication patterns and academic resilience. Social and Behavioural Sciences, 29, 87-90. https://doi.org/10.1016/j.sbspro.2011.11.210

Kanevsky, L., Corke, M., \& Frangkiser, L. (2008). The academic resilience and psychosocial characteristics of inner-city English learners in a museum-based school program. Education and Urban Society, 40(4), 452-475. https://doi.org/10.1177/0013124507304693

Kanner, A. D., Coyne, J. C., Schaefer, C., \& Lazarus, R. S. (1981). Comparison of two modes of stress management: Daily hassles and uplifts versus major life events. Journal of Behavioral Medicine, 4(1), 1-37. https://doi.org/10.1007/BF00844845

Kwok, O., Hughes, J. N, \& Luo, W., (2006). Role of resilient personality on lower achieving first grade students' current and future achievement. Journal of School Psychology, 45(1), 61-82. https://doi.org/10.1016/j.jsp.2006.07.002

La Foret, D. R., Watt, N. P., Diaz, L., McCullough, J., \& Barrrueco, S. (2000). Resilience and reading proficiency of Head Start graduates in inner-city schools. Paper presented at the annual Head Start research conference. https://doi.org/10.1002/pbc.24974

Ladd, R. J., Valrie, C. R., \& Walcott, C. M. (2014). Risk and resilience factors for grade retention in youth with sickle cell disease. Pediatric Blood Cancer, 61, 1252-1256. https://doi.org/10.1002/pbc.24974

Langenkamp, A. G. (2010). Academic vulnerability and resilience during the transition to high School: The role of social relationships and district context. Sociology of Education, 83(1), 1-19. https://doi.org/10.1177/0038040709356563 
Approaches to measuring academic resilience: A systematic review

Li, H., Martin, A. J., Armstrong, D., \& Walker, R. (2011). Risk, protection, and resilience in Chinese adolescents: A psycho-social study. Asian Journal of Social Psychology, 14(4), 269-282. https://doi.org/10.1111/j.1467-839X.2011.01356.X

Luthar, S. S., \& Cushing, G. (1999). Measurement issues in the empirical study of resilience: An overview. In M. Glanz, J. L. Johnson (Eds.), Resilience and development: Positive life adaptations (pp.129-160). New York, NY: Plenum.

Luthar, S. S., Cicchetti, D., \& Becker, B. (2000). The construct of resilience: a critical evaluation and guidelines for future work. Child Development, 71(3), 543-562. https://doi.org/10.1111/1467-8624.00164

Martin, A. J. \& Marsh, H. (2006). Academic resilience and its psychological and educational correlates: A construct validity approach. Psychology in the Schools, 43, 267-281. https://doi.org/10.1002/pits.20149

Martin, A. J. (2013). Academic buoyancy and academic resilience: Exploring 'everyday' and 'classic' resilience in the face of academic adversity. School Psychology International, 34(5), 488-500. https://doi.org/10.1177/0143034312472759

Martin, A. J. (2014). Academic buoyancy and academic outcomes: Towards a further understanding of students with attention-deficit/hyperactivity disorder (ADHD), students without ADHD, and academic buoyancy itself. British Journal of Educational Psychology, 84, 86-107. https://doi.org/10.1111/bjep.12007

Martin, A. J., \& Marsh, H. W. (2008). Academic buoyancy: Towards an understanding of students' everyday academic resilience. Journal of School Psychology, 46(3), 53-83. https://doi.org/10.1016/j.jsp.2007.01.002

Martin, A. J., Colmar, S. H., Davey, L. A., \& Marsh, H. W. (2010). Longitudinal modelling of academic buoyancy and motivation: Do the '5Cs' hold up over time? British Journal of Educational Psychology, 80, 473-496. https://doi.org/10.1348/000709910X486376

Martin, A. J., Yu, K., \& Hau, K.T. (2013). Motivation and engagement in the 'Asian Century': A comparison of Chinese students in Australia, Hong Kong, and Mainland China. Educational Psychology, 34(4), 417-439. https://doi.org/10.1080/01443410.2013.814199

Masten, A. S. (2001). Ordinary magic: Resilience processes in development. American Psychologist, 56(3), 227-238. https://doi.org/10.1037//0003-066X.56.3.227

Masten, A.S., Neeman, J., Andenas, S. (1994). Life events and adjustment in adolescents: The significance of event independence, desirability, and chronicity. Journal of Research on Adolescence, 4(1), 71-97. https://doi.org/10.1207/s15327795jra0401_5

Obradovic, J., Long, J. D., Cutili, J. J., Chan, C., Hinz, E., Heistad, D., \& Masten, A. (2009). Academic achievement of homeless and highly mobile children in an urban school district: Longitudinal evidence on risk, growth and resilience. Development and Psychopathology, 21(2), 493-518. https://doi.org/10.1017/S0954579409000273

Overstreet, S., \& Braun, S. (1999). A preliminary examination of the relationship between exposure to community violence and academic functioning. School Psychology Quarterly, 14(4), 380-396. https://doi.org/10.1037/h0089015

Peck, S. C., Roeser, R. W., Zarrett, N., \& Eccles, J. S. (2008). Exploring the roles of extracurricular activity quantity and quality in the educational resilience of vulnerable adolescents: Variable- and pattern-centered approaches. Journal of Social Issues, 64(1), 135-155. https://doi.org/10.1111/j.1540-4560.2008.00552.x

Perez, W., Espinoza, R., Ramos, K., Coronado, H., \& Cortes, R. (2009). Academic resilience among undocumented Latino students. Hispanic Journal of Behavioural Sciences, 31(2), 149-181. https://doi.org/10.1177/0739986309333020

Phan, H. P. (2016). Interrelations that foster learning: An investigation of two correlation studies. International Journal of Psychology, 5l(3), 185-195. https://doi.org/10.1002/ijop.12127

Pianta, R. C., \& Walsh, D. J. (1998). High risk children in schools: Constructing sustaining relationships. New York: Routledge.

Plunkett, S. W., Henry, C. S., Houltberg, B. J., Sands, T., \& Abarca-Mortensen, S. (2008). Academic support by significant others and educational resilience in Mexican-origin ninth grade students from intact families. 
Tudor, K. E., \& Spray, C. M.

Journal of Early Adolescence, 28(3), 333-355. https://doi.org/10.1177/0272431608314660

Putwain, D. W., \& Daly, A. L. (2013). Do clusters of test anxiety and academic buoyancy differentially predict academic performance? Learning and Individual Difference, 27(1), 157-162. http://dx.doi.org/10.1080/10615806.2011.582459

Raskauskas, J., Rubiano, S., Offen, I., \& Wayland, A. K. (2015). Do social self-efficacy and self-esteem moderate the relationship between peer victimisation and academic performance? Social Psychology Education, 18(2), 297-314. http://dx.doi.org/10.1007/s11218-015-9292-z

Reynolds, A. J. (1998). Resilience among black urban youth: Prevalence, intervention effects and mechanisms of influence. American Journal of Orthopsychiatry, 68(1), 84-100. https://doi.org/10.1037/h0080273

Rutter, M. (1985). Resilience in the face of adversity: Protective factors and resistance to psychiatric disorder. British Journal of Psychiatry, 147(6), 598-611. https://doi.org/10.1192/bjp.147.6.598

Rutter, M. (1987). Psychosocial resilience and protective mechanisms. American Journal of Orthopsychiatry, 57(3), 316- 331. https://doi.org/10.1111/j.1939-0025.1987.tb03541.x

Rutter, M. (2006). Implications of resilience concepts for scientific understanding. Annals New York Academy of Sciences, 1094, 1-12. http://dx.doi.org/10.1196/annals.1376.002

Samel, A. N., Sondergeld, T. A., Fischer, J. M., \& Patterson, N. C. (2011). The secondary school pipeline: Longitudinal indicators of resilience and resistance in urban schools under reform. The High School Journal, 94(3), 95-118. http://dx.doi.org/10.1353/hsj.2011.0005

Sarkar, M., \& Fletcher, D. (2013). How should we measure psychological resilience in sport performers? Measurement in Physical Education and Exercise Science, 17(4), 264-280. http://dx.doi.org/10.1080/1091367X.2013.805141

Sarwar, M., Inamullah, H., Khan, N., \& Anwar, N. (2010). Resilience and academic achievement of male and female secondary level students in Pakistan. Journal of College Teaching and Learning, 7(8), 19-24. https://doi.org/10.19030/tlc.v7i8.140

Schaufeli, W. B., Salanova, M., Gonzalez-Roma, V., \& Bakker, A. B. (2000). The measurement of engagement and burnout: A two sample confirmatory factor analytic approach. Journal of Happiness Studies, 3(1), 71-92. https://doi.org/10.1023/A:1015630930326

Schelbe, J. L., Franks, B. A., \& Miller, M. D. (2010). Emotion dysregulation and academic resilience in maltreated children. Child Youth Care Forum, 39(4), 289-303. https://doi.org/10.1007/s10566-010-9105-7

Schofield, K., \& Bates, L. (January, 2016). Nicky Morgan: True Grit. Politics Home. Retrieved from https://www.politicshome.com/news/uk/education/house/59358/nicky-morgan-true-grit

Sharkey, J. D., You, S., \& Schnoebelen, K. (2008). Relations among school assets, individual resilience, and student engagement for youth grouped by level of family functioning. Psychology in the Schools, 45(5). https://doi.org/402-418.10.1007/s10964-008-9360-1

Shumow, L. Vandell, D., \& Posner, J. (1999). Risk and resilience in the urban neighbourhood: Predictors of academic performance among low-income elementary school children. Merrill-Palmer Quarterly, 45(2), 309-331.

Simpson, R., Booth, J., Lawrence, M., Byrne, S., Mair, F., \& Mercer, S. (2014). Mindfulness-based interventions in multiple sclerosis: A systematic review. BMC Neurology, 14(15), 1-9. https://doi.org/10.1186/1471-2377-14-15

Skinner, E. A., \& Wellborn, J. G., (1997). Handbook of children's coping: linking theory and intervention. New York: Plenum Press.

Skinner, E., Pitzer, J., \& Steele, J. (2013). Coping as part of motivational resilience in school: A multidimensional measure of families, allocations, and profiles of academic coping. Educational and Psychological Measurement, 20(5), 1-33. https://doi.org/10.1177/0013164413485241

Smith, M. (2015). From adversity to buoyancy. The Psychologist, 28(9), 718-721.

Spencer, M. B., Cole, S. P., DuPree, D., Glymph, A., \& Pierre, P. (1993). Self-efficacy among urban African American early adolescents: Exploring issues of risk, vulnerability, and resilience. Development and Psychopathology, 5(4), 719-739. https://doi.org/10.1017/S0954579400006258 
Thornton, B., Collins, M., \& Daugherty, R. (2006). A study of resilience of American Indian high school students. Journal of American Indian Education, 45 (4), 4-16.

Trianes, M. V. Trianes, M. V., Blanca, M. J., Fernández-Baena, F. J., Escobar, M., \& Maldonado, E. F. (2011). IECI. Inventario de Estrés Cotidiano Infantil. Madrid, Spain: TEA Ediciones.

Ungar, M., \& Leibenberg, L. (2011). Assessing resilience across cultures using mixed methods: construction of the child and youth resilience measure. Journal of Mixed Methods Research, 5(2), 126- 149. https://doi.org/10.1177/1468017305058938

Von Secker, C. (2010). Science achievement in social contexts: analysis from national educational progress. The Journal of Educational Research, 98(2), 68-78. https://doi.org/10.3200/JOER.98.2.67-78

Wang, M., Haertal, G., \& Walberg, H. (1994). Educational resilience in inner cities. In M. Wang \& E. Gordon (Eds), Educational resilience in inner-city America: Challenges and prospects (pp. 45-72). Hillsdale, NJ: Lawrence Erlbaum Associates.

Waxman, H. C., Huang, S. L., \& Padron, Y. N. (1997). Motivation and learning environment differences between resilient and non-resilient Latino middle school students. Hispanic Journal of Behvioural Sciences, 19(2), 137-155. https://doi.org/10.1177/07399863970192003

Wayman, J. C. (2002). The utility of Educational Resilience as a framework for studying degree attainment in high school dropouts. Journal of Educational Research, 95(3), 167-178. https://doi.org/10.1080/00220670209596587

Windle, G., Bennett, K. M., \& Noyes, J. (2011). A methodological review of resilience measurement scales. Health and Quality of Life Outcomes, 9(8), 1-18. https://doi.org/10.1186/1477-7525-9-8

Woolley, M. E., \& Bowen, G. L. (2007). In the context of risk: Supportive adults and the school engagement of middle school students. Family Relations, 56(1), 92-104. https://doi.org/10.1111/j.1741-3729.2007.00442.x

Work, W. C., Cowen, E. L., Parker, G. R., \& Wyman, P. A. (1990). Stress resilient children in an urban setting. Journal of Primary Prevention, 11(1), 3-17. https://doi.org/10.1007/BF01324858 
Tudor, K. E., \& Spray, C. M. 